\title{
How to Understand MTrPs Induced Human Impairments and Dysfunctions?
}

\author{
Qiang Min Huang and Hui Zhang* \\ Department of Rehabilitation, Hudong Hospital, Shanghai China \\ *Corresponding author: Hui Zhang, Department of Rehabilitation, Hudong Hospital, Shanghai China
}

\begin{tabular}{|c|c|}
\hline ARTICLE INFO & ABSTRACT \\
\hline Received: 幽 March 11, 2019 & Citation: Qiang Min Huang, Hui Zhang. How to Understand MTrPs Induced Human Im- \\
\hline Published: March 20, 2019 & tions?. Biomed J Sci \& Tech Res 16(2)-2019. BJSTR. MS.ID.002812. \\
\hline
\end{tabular}

\section{Opinion}

Myofascial trigger point (MTrP) is a cytological lesion of skeletal muscle endplate. Under light microscopy, MTrPs' muscle fiber shape shows morphological changes of deep-stained large round in cross section and bead-like in longitudinal section, company with muscle spindle compression [1]. Rare mitochondria were found under electron microscopy. In biochemical and microdialysis studies, there was a high concentration of acetylcholine at the neuromuscular junction. The contractile nodules were palpated, which observed under B-mode ultrasonography [2]. The position of MTrPs existed spontaneous electrical discharges. Therefore, it is inferred that there may be an energy crisis at MTrPs, which may locally exude neurogenic substances that stimulate nerve endings, and then introduced into the spinal cord through afferent nerve and to the pain center of the brain to sense local pain through the upstream channel. However, when this sensation is transmitted to the spinal cord, it results in sensitization of the spinal cord, resulting in a characteristic of referred pain and local twitch response (muscle juming) at MTrPs.

Clinical observation shows that the referred pain has certain regularity, which is related to embryological development. In most cases, the closer to the MTrPs in human midline, the referred pain is the same position of the affected muscle. However, the MTrPs is far from human midline, the location of referred pain is often not in the affected muscle. Therefore, clinicians often locate the MTrPs according to the characteristics of referred pain in each muscle [3]. This is the fastest way to locate the MTrPs for acupuncture, and then confirm the accuracy of MTrPs in local twitch response (jumping) by needling. Most clinicians believe that induced jump is very important often with a good effect, but it is not very necessary depending on the experience of a clinician. With the development of MTrPs knowledge, the accumulation of clinical experience and the expansion of treatment scope, many clinicians have found that the treatment of pain, only from the occurrence of MTrPs, can only treat the simple fascia pain caused by the activity MTrPs, while the functional impairment of various parts of the human body, caused by the long-term multiple latent MTrPs, appears to be inadequate. Therefore, it is necessary to consider how the MTrPs can cause human dysfunction in different parts of the body. Here the following figure explains its possible pathogenesis Figure 1 shows that pathological factors that originate from primary MTrPs cause a disturbance of lever, fascia or ligament mechanics and coelomic capsular system and secondary MTrPs, leading to disorders of some part of the body or viscera [4]. Therefore, any treatment aimed at disorder adjustment is usually prone to recurrence or incomplete treatment if the source problem is not eliminated [5-6]. This kind of thinking suggests that when a clinician adjusts the lever and the fascial imbalance mechanics and the disturbance of the coelomic capsular system, he should also consider the primary MTrPs at the source of treatment, so as to cure the patient's disease better. 


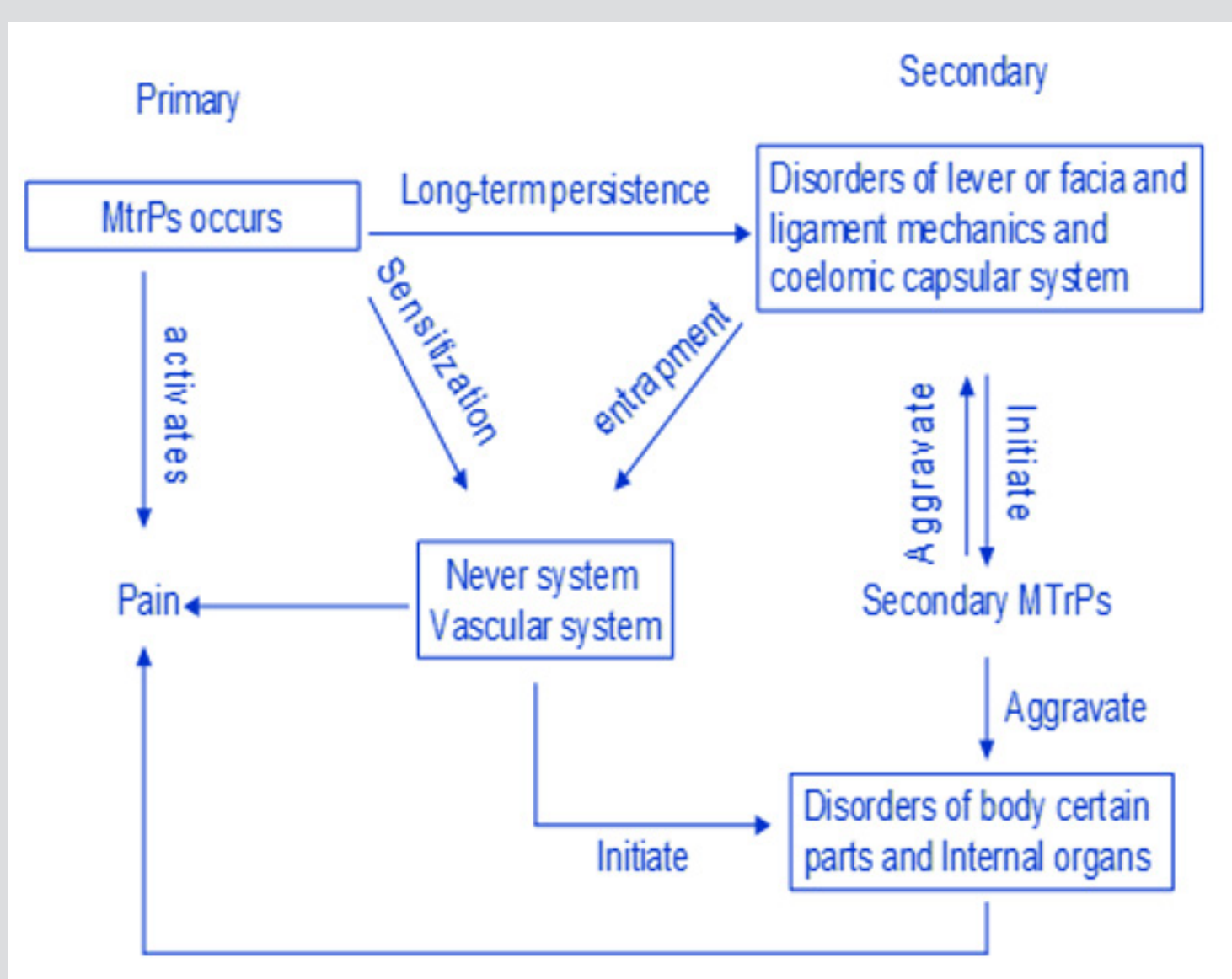

Figure 1: The diagram of various disorders and impairments which relate to MTrPs.

\section{References}

1. Huang QM, Ye G, Zhao ZY, Lv JJ, Tang L (2013) Myoelectrical activity and muscle morphology in a rat model of myofascial trigger points induced by blunt trauma to the vastus medialis. Acupunct Med 31(1): 65-73.

2. Huang QM, Lv JJ, Ruanshi QM, Liu L (2015) Spontaneous electrical activities at myofascial trigger points at different stages of recovery from injury in a rat model. Acupunct Med 33(4): 319-24.

3. Liu QG, Liu L, Huang QM, Nguyen TT, Ma YT, et al. (2017) Decreased Spontaneous Electrical Activity and Acetylcholine at Myofascial Trigger Spots after Dry Needling Treatment: A Pilot Study. Evid Based Complement Alternat Med.

\section{ISSN: 2574-1241}

DOI: 10.26717/BJSTR.2019.16.002812

Hui Zhang. Biomed J Sci \& Tech Res

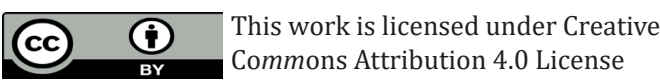

Submission Link: https://biomedres.us/submit-manuscript.php
4. Zhang H, Lü JJ, Huang QM, Liu L, Liu QG, et al. (2017) Histopathological nature of myofascial trigger points at different stages of recovery from injury in a rat model. Acupunct Med 35(6): 445-451.

5. Zhuang X, Tan S, Huang Q (2014) Understanding of myofascial trigger points. Chin Med J 127(24): 4271-4277.

6. Huang QM, Zhang YD, Ma YT (2018) Understanding of Myofascial Trigger Points: Debates between acupuncture and dry needling (Chinese). Chin Acupunct 5(3): 1146.

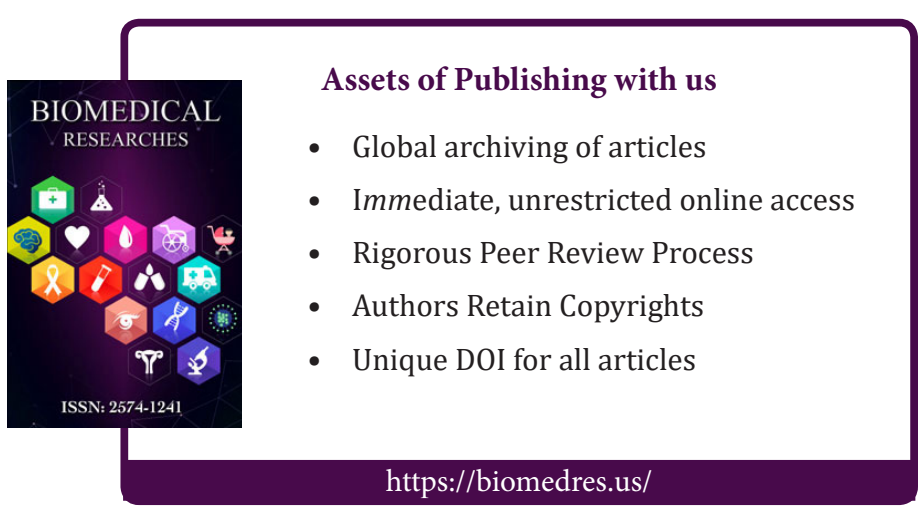

